\title{
Corrigendum
}

\section{An adaptive guidance meta-heuristic for the vehicle routing problem with splits and clustered backhauls}

\author{
Michela Lai, Maria Battarra, Massimo Di Francesco and Paola Zuddas
}

Journal of the Operational Research Society (2015) 66(7), 1236. doi:10.1057/jors.2015.12

Published online 29 April 2015

Correction to: Journal of the Operational Research Society (2014). doi:10.1057/jors.2014.123

In the above paper Figure 1 is incorrectly referred to in Section 5.4, the text should have referred to Figure 2. In addition Figure 2 was missing from the paper. Two typos occurred in the comparison between $Q=6$ and $Q=1$. The corrected text and Figure 2 are shown below.

\subsection{Implications for the case study}

Grendi Trasporti Marittimi is interested in converting its fleet of vehicles in solely trucks able to carry two containers each. Therefore, it is important to determine which transportation costs will be paid according to the new fleet configuration. The quantification of routing costs is performed by the proposed meta-heuristic, which compares two scenarios for this analysis:

$\mathrm{Q}=1$, that is, all vehicles carry one container;

$\mathrm{Q}=2$, that is, all vehicles carry two containers.

In addition, transportation capacities could be further increased by LCVs, if allowed. Therefore, we carry out a prospective study on LCVs, whose transportation capacity $Q$ is taken for granted from existing studies. In this stage, two additional scenarios are considered to determine how transportation capacities are linked to routing costs:

$\mathrm{Q}=4$, that is, all vehicles carry four containers;

$\mathrm{Q}=6$, that is, all vehicles carry six containers.

Results are reported in Figure 2, which shows the link between capacities and costs for instances with 10, 20, 30, 40 and 50 customers. More precisely:

- If one considers the instances with $\mathrm{Q}=2$ with respect to those with $\mathrm{Q}=1$, the routing cost decreases by $44.30 \%$ in the case of 10 customers, up to $47.65 \%$ in the case of 50 customers.

- If one compares the instances with $Q=4$ to those with $\mathrm{Q}=2$, the routing cost decreases by $38.72 \%$ in the case of 10 customers, up to $46.06 \%$ in the case of 40 customers.

- If one considers the instances with $\mathrm{Q}=6$ with respect to those with vehicles $\mathrm{Q}=4$, the routing cost decreases by $20.02 \%$ in the case of 10 customers, up to $26.94 \%$ in the case of 20 customers.

Note that the marginal improvement in case of $Q=6$ with respect to $Q=4$ is relatively small, but if one considers the instances with $\mathrm{Q}=6$ with respect to those with $\mathrm{Q}=1$, the routing cost decreases by $72.70 \%$ in the case of 10 customers and up to $78.63 \%$ in the case of 50 customers.

The most likely scenario for this case study is the introduction of the third container per truck, because this option already meets the European regulations on LCVs. Figure 2 shows that this scenario is expected to produce savings ranging between 20 and $25 \%$ with respect to the case $\mathrm{Q}=2$.

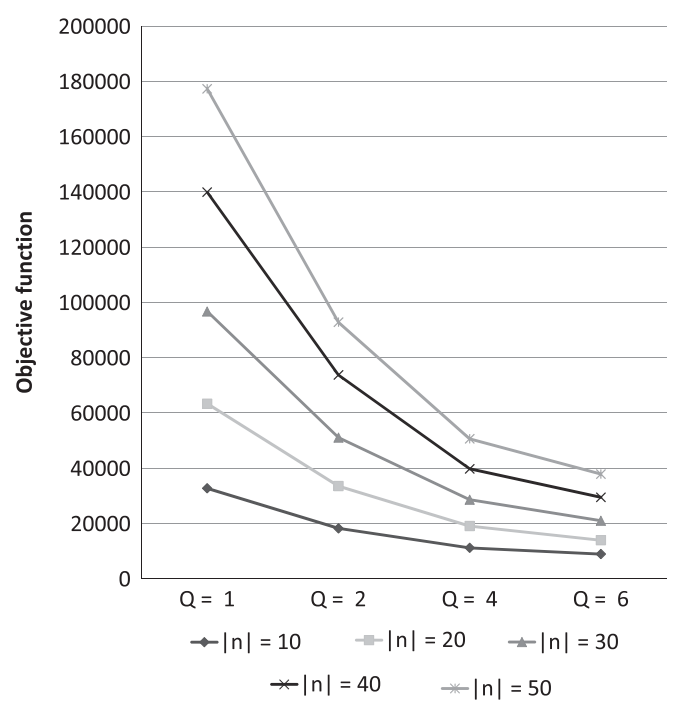

Figure 2 Efficiency of the distribution with larger transportation capacities. 